\title{
Anomalous Behavior of the Near-Threshold Photoionization Cross Section of the Neon Isoelectronic Sequence: A Combined Experimental and Theoretical Study
}

\author{
H. S. Chakraborty, ${ }^{1}$ A. Gray, ${ }^{2}$ J. T. Costello, ${ }^{2}$ P. C. Deshmukh, ${ }^{1}$ G. N. Haque ${ }^{3}$ E. T. Kennedy, ${ }^{2, *}$ \\ S. T. Manson, ${ }^{4}$ and J-P. Mosnier ${ }^{2}$ \\ ${ }^{1}$ Department of Physics, Indian Institute of Technology, Madras 600036, India \\ ${ }^{2}$ Centre for Laser Plasma Research, School of Physical Sciences, Dublin City University, Dublin 9, Ireland \\ ${ }^{3}$ Department of Physics, Morehouse College, Atlanta, Georgia 30314 \\ ${ }^{4}$ Department of Physics and Astronomy, Georgia State University, Atlanta, Georgia 30303
}

(Received 24 May 1999)

\begin{abstract}
We present a combined theoretical and experimental investigation of photoionization along the $\mathrm{Ne}$ isoelectronic sequence and show that the near-threshold behavior of the cross section for $\mathrm{Si}^{4+} \mathrm{differs}$ radically from the nearby ions in the sequence. We demonstrate that the general nature of the underlying physics implies that dramatic changes in near-threshold behavior may be expected for many other ions.
\end{abstract}

PACS numbers: $32.80 . \mathrm{Fb}, 32.80 . \mathrm{Hd}, 52.25 . \mathrm{Qt}$

The response of positive ions to ionizing radiation, the fundamental process of photoionization, is a dominant process in the universe. However, it is largely unexplored experimentally due to the difficulty of producing and maintaining appropriate densities of multiply charged ions, along with the requirement for high-flux sources in the extreme UV and $\mathrm{x}$-ray ranges [1,2]. Photoionization is particularly attractive for theoretical interpretation due to (i) selectivity, owing to dipole selection rules, (ii) the fact that the photon disappears, thereby simplifying the final state, and (iii) the weak coupling between the photon and target electrons, allowing focus on the many-body aspects of the electron-electron interactions of the target ion [3-5].

From a fundamental point of view, positive ions provide a "laboratory" to investigate the dynamic interplay between many-body electron-electron correlations, electron-nucleus interactions, and relativistic effects by looking along an ionic sequence. With increasing ionization, as the electron-nucleus interactions dominate, the photoionization behavior will tend towards hydrogenic; however, this trend towards simplicity is not at all monotonic, and very significant changes in the cross section behavior may occur for particular ions. In this Letter, we report the first combined theoretical and experimental investigation of photoionization along the $\mathrm{Ne}$ isoelectronic sequence and show that the near-threshold behavior of the cross section for $\mathrm{Si}^{4+}$ differs radically from the nearby ions in the sequence. We demonstrate that the general nature of the underlying physics implies that dramatic changes in near-threshold behavior may be expected for many other ions.

Photoionization of the $\mathrm{Ne}$ isoelectronic sequence, aside from its importance as the response of the simplest noble gas structure with multiple shells $\left(1 s^{2} 2 s^{2} 2 p^{6}\right)$ to ionizing radiation, is also of particular interest owing to the significant cosmic abundance of $\mathrm{Ne}, \mathrm{Na}^{+}, \mathrm{Mg}^{2+}$, $\mathrm{Al}^{3+}, \mathrm{Si}^{4+}, \mathrm{S}^{6+}, \mathrm{Ar}^{8+}, \mathrm{Ca}^{10+}$, and $\mathrm{Fe}^{16+}$ [6]. Furthermore, the study of photoionization provides information on the inverse processes of radiative and dielectronic recombination which are of importance in modeling astrophysical [7] and laboratory [8] plasmas, particularly for low-energy electrons corresponding to the near-threshold region, which is the focus of this Letter.

Following the pioneering work of Codling et al. [9], several extensive experimental investigations on neutral $\mathrm{Ne}$ have been carried out, aided by the relative simplicity of working with a gas and recent developments in storage ring sources [10]. For ions of the Ne sequence, previous experimental results are, however, very sparse. Photoabsorption data for $\mathrm{Na}^{+}$has been reported from a resonant laser-driven ionization experiment [11]; $\mathrm{Mg}^{2+}$ was studied using a twin-spark method [12], and $\mathrm{Mg}^{2+}$ and $\mathrm{Al}^{3+}$ resonance features were also recorded [13]. All data to date for ions in this sequence were recorded photographically and, particularly for $\mathrm{Al}^{3+}$, were masked by emission features.

The extensive theoretical work on neutral Ne includes a number of accurate calculations using a variety of methodologies, e.g., $R$ matrix [14] and relativistic-random-phase approximation (RRPA) [15,16]. Calculations have been carried out as part of the Opacity Project [17] for the ions of the Ne sequence based on nonrelativistic $R$-matrix theory. Calculations on Ne-like Fe have been performed [18] which show substantial differences with the nonrelativistic result, indicating the increasing importance of relativistic effects along the sequence. The relativistic result agrees quite well with a more recent RRPA calculation [19].

Our measurements on the photoabsorption thresholds for the $\mathrm{Ne}$ sequence up to $\mathrm{Si}^{4+}$ were carried out using the dual laser plasma (DLP) technique [2]. The output of a $\mathrm{Nd}$ :YAG laser ( $\sim 1 \mathrm{~J}$ in $10 \mathrm{~ns})$ was focused tightly onto a tungsten surface in vacuo, to form a small hot dense plasma which served as a short-pulse backlighting source of vacuum ultraviolet continuum radiation. A second synchronized Nd:YAG laser (pulse $\sim 0.3 \mathrm{~J}$ in $10 \mathrm{~ns}$ ) 
generated an absorbing plasma plume situated on the optical axis between the backlighting plasma and the entrance slit of the spectrometer. By appropriate choices of target material (solid $\mathrm{Na}, \mathrm{Mg}, \mathrm{Al}$, or $\mathrm{Si}$, respectively), laser irradiation conditions and the interplasma time delay, we optimized the plasma conditions to maximize the Ne-like ion population and recorded the near-threshold behavior for each isoelectronic ion in turn. Using the backlighting Nd:YAG laser alone, the background continuum spectrum $\left(I_{0}\right)$ was obtained. By firing both lasers, the transmitted intensity $(I)$ was recorded. The quantity $\ln \left(I_{0} / I\right)$ was subsequently computed to generate the required photoabsorption data. Signal averaging was employed to improve the signal-to-noise ratio of both $I$ and $I_{0}$. Deconvolution techniques were applied to enhance the effective spectral resolution by a factor of about 2 . The interplasma delay and laser pulse profiles were monitored on every shot to ensure reproducible conditions. A notable advantage of the present study was that all of the photoabsorption spectra were recorded with the same experimental setup.

Our calculations were performed using the RRPA plus multichannel quantum defect theory $[15,16]$, which agrees well with experiment and other sophisticated theoretical methodologies for Ne. The calculations are expected to become even more accurate along the sequence, because multielectron interactions, which arise from the part of the Hamiltonian being approximated, become less important with increasing ionization. All members of the $\mathrm{Ne}$ sequence have been considered, up to $Z=100$. Each of the seven relativistic single excitation channels arising from the $2 s$ and $2 p$ subshells were included: $2 s \rightarrow \varepsilon p_{1 / 2}$, $\varepsilon p_{3 / 2} ; 2 p_{1 / 2} \rightarrow \varepsilon s_{1 / 2}, \varepsilon d_{3 / 2} ; 2 p_{3 / 2} \rightarrow \varepsilon s_{1 / 2}, \varepsilon d_{3 / 2}, \varepsilon d_{5 / 2}$. Since we are interested in the behavior of the cross section near threshold, it is unlikely that the omission of the $1 \mathrm{~s}$ channels will have an appreciable effect; test calculations confirmed this point.

The results of the calculations for the cross section at the $2 s^{2} 2 p^{52} P_{1 / 2}$ threshold are shown in Table I (column 3 ) and Fig. 1 (solid points). The outstanding feature is the smooth evolution of the cross section along the sequence, with the exception of the $\mathrm{Si}^{4+}$ results which are smaller by more than an order of magnitude. Also shown in Table I (column 2) and Fig. 1 (continuous line) are the calculated results with the coupling between $2 s$ and $2 p$ channels omitted, and a marked difference from the full RRPA result is seen. Without the coupling, the threshold $2 p$ cross section for $\mathrm{Si}^{4+}$ falls into the pattern established by the neighboring members of the isoelectronic sequence, while the other members of the sequence are substantially the same. Thus, the threshold behavior anomaly for $\mathrm{Si}^{4+}$ is related to the coupling between $2 s$ and $2 p$ channels.

To portray the situation more clearly, the cross section just above the ${ }^{2} P_{1 / 2}$ threshold is shown in Fig. 2 for photoionization of $\mathrm{Na}^{+}, \mathrm{Mg}^{2+}, \mathrm{Al}^{3+}, \mathrm{Si}^{4+}$, and $\mathrm{P}^{5+}$. For $\mathrm{Na}^{+}$, the remnants of the $2 p \rightarrow \varepsilon d$ delayed maximum, evident for neutral neon, is responsible for the slightly
TABLE I. Calculated photoionization cross section (MB) at the ${ }^{2} P_{1 / 2}$ threshold for the first six members of the neon isoelectronic sequence without ( 5 channel) and with ( 7 channel) coupling between the $2 p$ and $2 s$ ionization channels.

\begin{tabular}{lcc}
\hline \hline Ion & 5 Channel & 7 Channel \\
\hline $\mathrm{Ne}$ & 5.61 & 5.77 \\
$\mathrm{Na}^{+}$ & 6.98 & 7.24 \\
$\mathrm{Mg}^{2+}$ & 6.21 & 6.49 \\
$\mathrm{Al}^{3+}$ & 4.93 & 5.27 \\
$\mathrm{Si}^{4+}$ & 3.79 & 0.30 \\
$\mathrm{P}^{5+}$ & 2.91 & 2.90 \\
\hline \hline
\end{tabular}

increasing cross section at threshold; most of the rise has moved below threshold into the discrete region. $\mathrm{Mg}^{2+}$ shows an almost flat cross section with a slight increase well above threshold. The $\mathrm{Al}^{3+}$ cross section is almost flat at threshold but is gradually dominated by the inner-shell $2 s \rightarrow 3 p$ resonance which has moved down in energy to within $\sim 13 \mathrm{eV}$ of the threshold; the analogous resonance in $\mathrm{Mg}^{2+}$ explains the slight tailing up of the cross section seen in Fig. 2(c). At $\mathrm{Si}^{4+}$, this resonance just straddles the ionization limit, thereby changing the threshold cross section dramatically. For $\mathrm{P}^{5+}$, the $2 s \rightarrow 3 p$ resonance has moved well below the ionization limit and the threshold cross section behavior has returned to "normal." However, the $2 s \rightarrow 4 p$ resonance is now seen to move down into the near-threshold region. The movement of successive innershell resonances through the ionization limit evidently occurs along the entire neon sequence.

The key, therefore, to the anomalous threshold cross section is clearly the fact that the inner shell $2 s \rightarrow n p$ resonances move into the discrete region, below the $2 p$ thresholds, with increasing $Z$ along the sequence. As $Z \rightarrow \infty$, for any isoelectronic sequence the $2 s$ and $2 p$

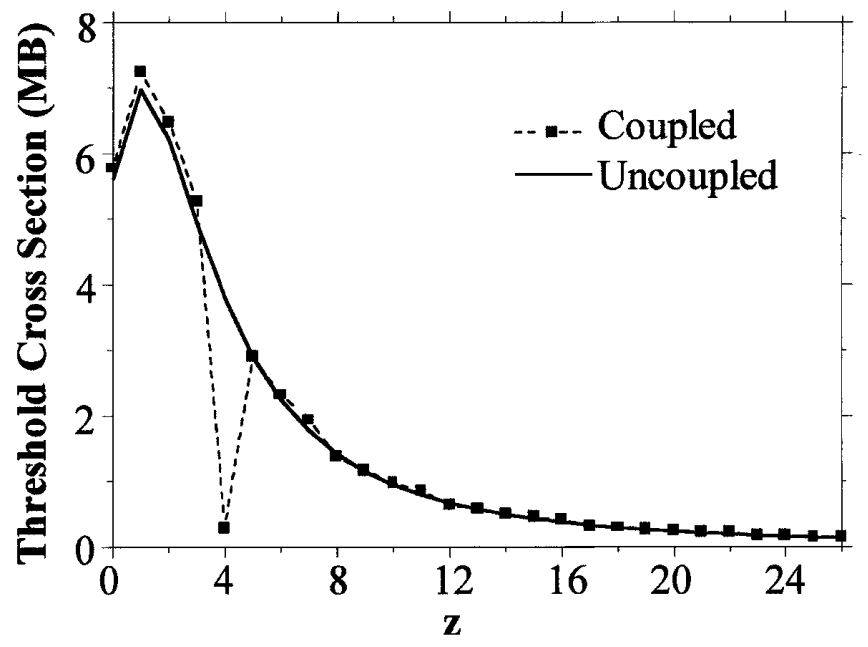

FIG. 1. Calculated photoionization cross section (MB) at the ${ }^{2} P_{1 / 2}$ threshold vs initial state charge $z$ for the neon isoelectronic sequence. Results are given with and without inclusion of the coupling between the $2 s$ and $2 p$ channels. 


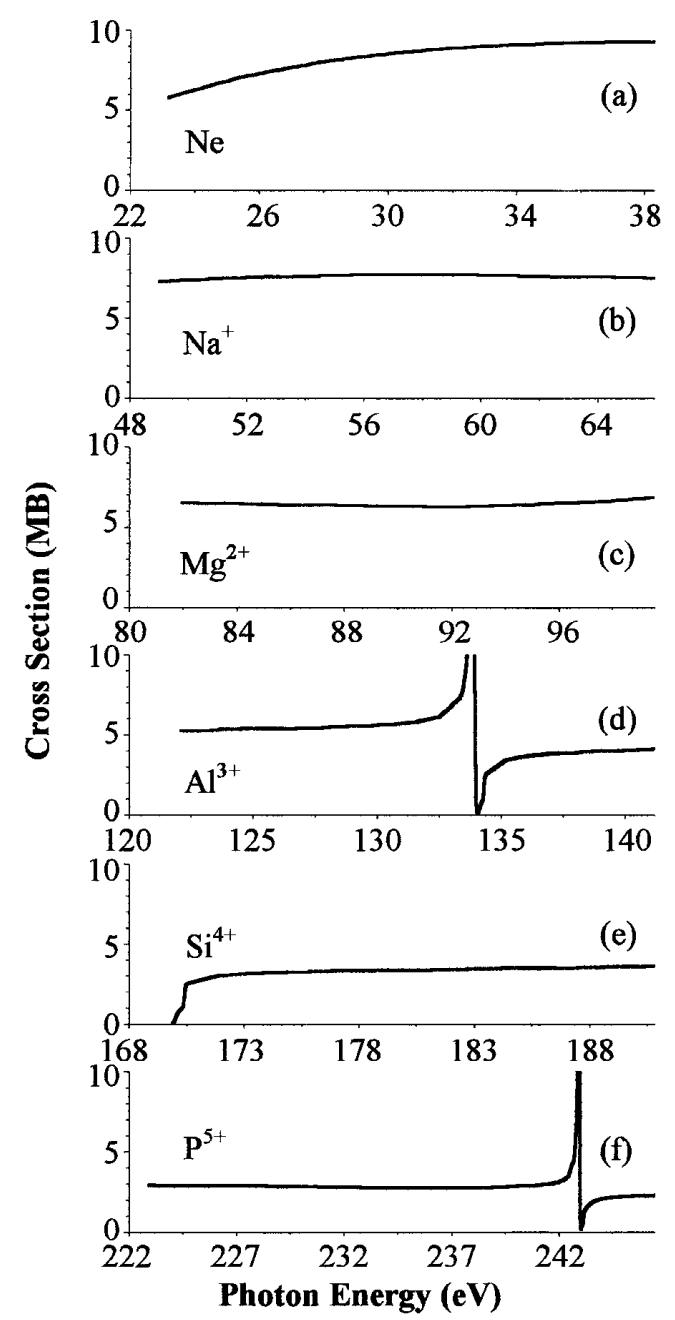

FIG. 2. Calculated near-threshold photoionization cross sections for (a) $\mathrm{Ne}$, (b) $\mathrm{Na}^{+}$, (c) $\mathrm{Mg}^{2+}$, (d) $\mathrm{Al}^{3+}$, (e) $\mathrm{Si}^{4+}$, and (f) $\mathrm{P}^{5+}$. Note that the full height of the $\mathrm{Al}^{3+}$ resonance peak is not shown.

thresholds become degenerate since they have the same principal quantum number. The downward movement of inner-shell resonances with respect to outer-shell thresholds therefore occurs for all sequences for which the outermost subshell is other than $\ell=0$; this constitutes the vast bulk of the periodic table. Whenever an inner-shell resonance, on its downward trek along the sequence, falls in close proximity to an outer threshold, consequent dramatic modification of the near-threshold cross section can result. Therefore, this resonance-induced threshold effect is quite a general phenomenon.

In Fig. 3 we show a comparison for $\mathrm{Na}^{+}$between the calculated photoionization cross section above threshold and the measured DLP deconvolved photoabsorption spectrum. For the comparison, the theoretical curve, which used simple Dirac-Fock thresholds, is shifted by $-3.8 \mathrm{eV}$ in order to match the energy of the second resonance with that of the experiment. Generally, good agreement in terms of the resonance positions and pro-

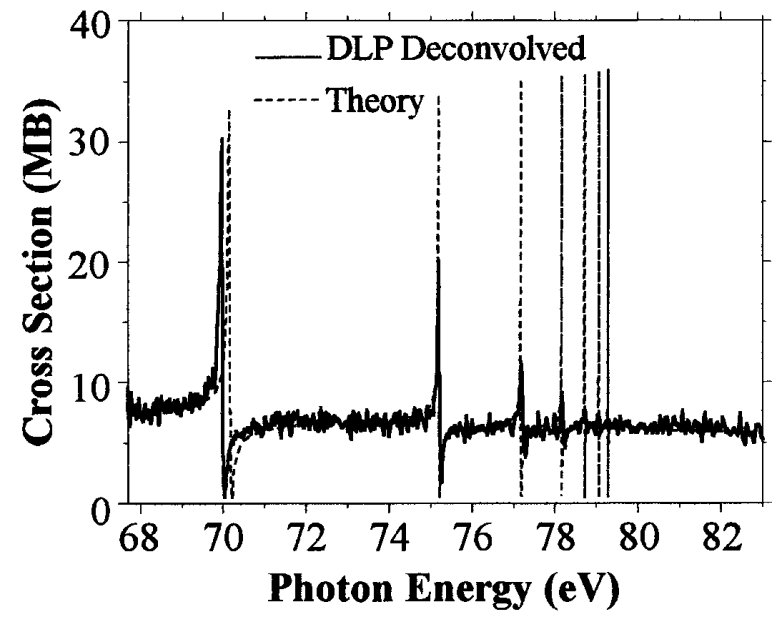

FIG. 3. Comparison between the (deconvolved) measured relative total photoabsorption cross section of $\mathrm{Na}^{+}$, in the region of the $n p$ inner-shell resonances, with the calculated photoionization cross section. The experimental result is normalized to the theory in the background (nonresonant) region.

files is observed. For the lowest lying resonance the profile agrees very well; the limited spectral resolution reduces the contrast of the experimentally observed higher resonances. Figure 4 shows our DLP recorded results for the sequence members $\mathrm{Na}^{+}$through $\mathrm{Si}^{4+}$ in the threshold energy regions. For the ions $\mathrm{Na}^{+}$to $\mathrm{Al}^{3+}$ we see the valence transitions $(2 p \rightarrow n s, n d)$ running to the series limits indicated, with a smooth continuation in the cross section across the limits. For $\mathrm{Si}^{4+}$ the behavior is strikingly different. The near-threshold cross section is now dominated by the inner-shell $2 s \rightarrow 3 p$ resonance which has moved down to just below the ionization limit. Detailed measurements show that the resonance is centered at $165.4 \mathrm{eV}$, whereas the photoionization limits ${ }^{2} P_{3 / 2}$ and ${ }^{2} P_{1 / 2}$, as determined from extrapolation of the valence $2 p \rightarrow n s, n d$ series, lie at the indicated positions of 166.7 and $167.2 \mathrm{eV}$, respectively. The experimental results, which confirm the presence of the $2 s \rightarrow 3 p$ resonance just below the ionization limit, therefore support the theoretical calculations indicating the anomalous behavior of the near-threshold cross section for $\mathrm{Si}^{4+}$.

A final point needs to be made regarding the experimental data for $\mathrm{Si}^{4+}$. For an inner-shell resonance above the photoionization limit, an asymmetric Fano profile may be expected due to the interaction between the quasidiscrete resonance state and the associated continuum states [3]. For $\mathrm{Si}^{4+}$ the photoionization limit, determined from the extrapolation of the valence series, lies a full $\mathrm{eV}$ above the energy of the inner-shell $3 p$ resonance, and so the profile of the resonance is at first sight surprising. However, in a plasma environment the microfields depress the ionization limit relative to that of totally free ions. For $\mathrm{Si}^{4+}$ therefore, the near coincidence of the limit and the $3 p$ resonance makes forced 


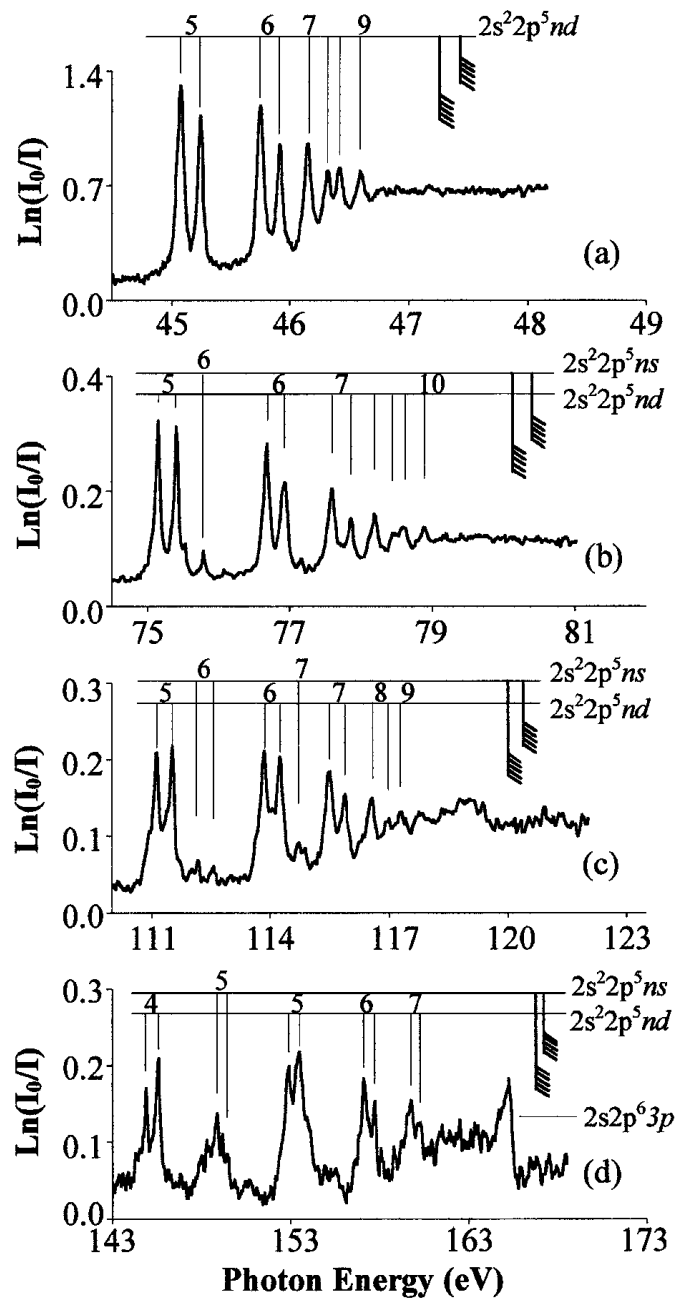

FIG. 4. Experimental photoabsorption spectra for (a) $\mathrm{Na}^{+}$, (b) $\mathrm{Mg}^{2+}$, (c) $\mathrm{Al}^{3+}$, and (d) $\mathrm{Si}^{4+}$ with many of the $2 s^{2} 2 p^{5} n l$ absorption features identified. The $2 s^{2} 2 p^{52} P_{3 / 2,1 / 2}$ thresholds are indicated at the upper right of each figure. Note the changes in the energy scales along the sequence.

autoionization possible, providing an explanation for the experimental profile. Indeed, this near coincidence makes the case of $\mathrm{Si}^{4+}$ an interesting one for future studies on the interaction of autoionizing states through variation of the plasma conditions.

In conclusion, both experimental and theoretical results show that the photoionization cross section of the $\mathrm{Ne}$ isoelectronic sequence in the threshold region exhibits a dramatic anomaly at $\mathrm{Si}^{4+}$, a phenomenon that was traced to the moving of inner-shell resonances below threshold with increasing $Z$. It was further pointed out that this should be a general phenomenon throughout the periodic system. Finally, the results suggest strongly that interpolation or extrapolation of photoionization and recombination cross sections along sequences should be viewed with extreme caution unless the role of inner-shell transitions is accurately included.

This work was supported by the National Science Foundation, NASA, and Enterprise Ireland.

*Electronic address: eugene.kennedy@dcu.ie

[1] F. J. Wuilleumier and J.B. West, in $V U V$ and Soft X-Ray Photoionization, edited by U. Becker and D. A. Shirley (Plenum, New York, 1996), p. 561, and references therein.

[2] E. T. Kennedy et al., Opt. Eng. 33, 3964 (1994); E. T. Kennedy, J. T. Costello, and J-P. Mosnier, in Atomic and Molecular Physics, edited by I. Alvarez, C. Cisneros, and T. J. Morgan (World Scientific, Singapore, 1995), p. 495, and references therein.

[3] U. Fano and J. W. Cooper, Rev. Mod. Phys. 41, 441 (1968).

[4] A.F. Starace, in Handbuch der Physik, edited by W. Melhorn (Springer-Verlag, Berlin, 1982), Vol. 31, p. 1.

[5] M. Ya. Amusia, Atomic Photoeffect (Plenum, New York, 1990).

[6] A. Hibbert and M.P. Scott, J. Phys. B 27, 1315 (1994).

[7] J. M. Schull, Phys. Scr. T47, 165 (1993).

[8] Atomic Processes and Plasmas, edited by E. Oaks and M. S. Pindzola (AIP, Woodbury, NY, 1998).

[9] K. Codling, R. P. Madden, and D. L. Ederer, Phys. Rev. 155, 26 (1967).

[10] K. Schultz et al., Phys. Rev. A 54, 3095 (1996).

[11] T. B. Lucatorto and T. J. McIlrath, Phys. Rev. Lett. 37, 428 (1976).

[12] J. M. Esteva and G. Mehlman, Astrophys. J. 193, 747 (1974).

[13] S. O. Kastner et al., Phys. Rev. A 16, 577 (1977).

[14] N. S. Scott and P. G. Burke, J. Phys. B 13, 4299 (1980), and references therein.

[15] W. R. Johnson et al., Phys. Scr. 21, 409 (1980).

[16] W. R. Johnson and K. T. Cheng, Phys. Rev. A 20, 978 (1979).

[17] W. C. Mendoza, Phys. Scr. T65, 198 (1996).

[18] M. Mohan et al., Phys. Rev. A 57, 3489 (1998).

[19] N. Haque et al. (to be published). 